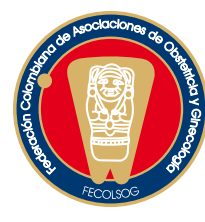

\title{
Editorial
}

\section{TABAQUISMO EN EL EMBARAZO: UNA EPIDEMIA SILENCIOSA PREVENIBLE EN COLOMBIA}

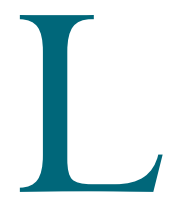

a Organización Mundial de la Salud (OMS) considera que el tabaco es una de las primeras causas de enfermedad crónica y de muerte prevenible en el mundo. En pacientes obstétricas, la exposición a los productos del tabaco es la causa prevenible más importante asociada con el retardo del crecimiento intrauterino ( $\mathrm{RCIU})$, el parto pretérmino y el bajo peso al nacer. Se ha asociado además, con muerte fetal intrauterina y con anormalidades en la función placentaria. En el período neonatal se asocia con el síndrome de muerte súbita neonatal, infecciones como otitis y neumonía, y enfermedades crónicas como el asma y la obesidad. ${ }^{1,2}$

El consumo de tabaco compromete todas las edades, pero de manera preocupante se aprecia aumento en poblaciones susceptibles como son los adolescentes y personas jóvenes. A nivel mundial, las niñas y las mujeres son actualmente blanco específico de la industria del tabaco, en la promoción indiscriminada del consumo del tabaco y cigarrillos. ${ }^{3}$ Es muy grave que en encuestas globales se haya demostrado un aumento en el consumo de cigarrillos y tabaco en niñas entre los 13-15 años de edad. Sin embargo, este aumento en el consumo no ha afectado a todos los países de igual manera. Los países de altos ingresos, según la clasificación de países por ingresos del Banco Mundial, han visto una reducción en el consumo del tabaco, mientras que en países clasificados como de bajos, o medio/altos ingresos como el nuestro, el consumo se ha elevado. ${ }^{3}$ En los países de economías de bajo ingreso se calcula que el 9\% de las mujeres fuman. Las mujeres jóvenes cada vez más se exponen a este flagelo, y en nuestro país una de cada cuatro adolescentes se embaraza.

(http://www.who.int/tobacco/resources/ publications/tobacco_atlas/en/index.html).

Se han utilizado múltiples estrategias para disminuir el consumo de tabaco; entre ellas se encuentra la creación de impuestos para aumentar el costo de los cigarrillos, esta medida en nuestro medio ha tenido un impacto limitado debido al flagelo del contrabando. Sin embargo, se han dado pasos en la dirección correcta con la aprobación, en Colombia, de la Ley 1335 de julio 21 de 2009 o Ley integral de control de tabaco, quince meses después de que Colombia ratificara el convenio marco para el control del tabaco de la Organización Mundial de la Salud.

La prevalencia del tabaquismo en la gestante en nuestro país no es conocida con exactitud, a pesar de que el hábito de fumar es considerado un evento de vigilancia en salud pública. Su efecto en el embarazo ha sido poco evaluado, y no se le reconoce al problema la importancia que requiere. Pilar VélezGómez, y colaboradores de la Universidad Pontificia Bolivariana de Medellín, en un estudio descriptivo y publicado en esta revista en el año 2006, encontraron en su población de influencia que el 17\% de los nacidos vivos tenían bajo peso al nacer, 21\% fueron pretérmino y 12\% tenían RCIU. Ellos informan una asociación significativa entre el bajo peso al nacer y el tabaquismo (RR 1,95 IC 95\% 1,19-3,17). ${ }^{4}$

En este número de la revista, Hernán CortésYepes, publica el reporte de la prevalencia de 
tabaquismo en 2171 gestantes consecutivas, evaluadas en la unidad de Medicina Fetal del Hospital Universitario San Vicente de Paúl Fundación, Institución de referencia ubicada en la ciudad de Medellín. La metodología utilizada fue la administración de una encuesta por parte del autor, quien reconoce las limitaciones de la metodología utilizada. Cortés-Yepes reporta una prevalencia de 7,3\% que corresponde a 160 gestantes. De estas, $70(43,7 \%)$ continuaron fumando durante el embarazo en promedio 7,2 cigarrillos/día. Aunque el estudio no describe si se utilizó consejería a las participantes para la cesación del consumo de tabaco; y no se incluyó en el estudio el período posparto, cuando la mayoría de gestantes reinciden en el tabaquismo, tiene un carácter innovador, ya que no existen reportes previos o contemporáneos de esta población en el país.

Reportes de otros países latinoamericanos indican que la prevalencia es variable, siendo tan alta como el $18 \%$ o tan baja como el $0,6 \%$ de las gestantes. ${ }^{5}$ La prevalencia reportada por Cortés-Yepes se puede considerar intermedia, sin embargo, no menospreciable dadas las características de la población asistida en ese centro de alta complejidad para la región. Bajo estas condiciones, hay que tener en cuenta que las fumadoras tienden a no reportar el uso del tabaco en el embarazo, con mayor razón si tienen factores de riesgo identificados. Es recomendable que en futuros estudios epidemiológicos, se consideren estas preguntas como ineludibles, además el uso de marcadores biológicos para verificación de la exposición, como la cotinina en orina o saliva, el monóxido de carbono exhalado, que son de fácil disponibilidad y registro. ${ }^{6}$ Se requieren estudios que evalúen la exposición durante el embarazo al tabaco y otros tóxicos; que tengan en cuenta las condiciones sociales, culturales y económicas de la población colombiana y latinoamericana.

El período prenatal es una oportunidad de oro para la identificación de la exposición activa y pasiva a los productos del tabaco. En Colombia más del 80\% de las instituciones administradoras y prestadoras de los servicios de salud utilizan el carné perinatal del Centro Latinoamericano de Perinatología (CLAP), con algunas modificaciones en algunas de ellas. Este instrumento incluye el hábito de fumar como factor de riesgo, tanto como consumidora activa como pasiva. Lamentablemente esta información tiene problemas en cuanto a la calidad de los registros, hay subregistro y falta de continuidad en los datos. Se requiere del análisis de esta información para consolidar los diagnósticos y medir el impacto de la morbilidad directa en el feto.

Por otra parte, es responsabilidad de los agentes de salud, no solo la prestación de servicios, también la vigilancia de los eventos de salud pública, así como la promoción de la salud; de esta manera la búsqueda de la exposición al tabaco se deberá iniciar desde la fase preconcepcional. Sin embargo, no existe la política ni la cultura apropiada de promoción de la salud en mujeres en edad reproductiva. La prevención del tabaquismo debe ser tema de la agenda pública y del sector educativo.

Existen estudios que refieren una alta prevalencia del tabaquismo entre los agentes de salud, aún desde las fases tempranas de la formación profesional. Esto sugiere una preocupante falta de correspondencia del personal de salud con su compromiso y protección de la salud de las pacientes y la propia. Mejía y colaboradores reportaron en Argentina, un uso actual de tabaco en el 35\% de 235 obstetras ginecólogos encuestados en el 2006.

Esta situación requiere la reorientación de los currículos de las facultades de salud, así como la implementación de estrategias soportadas por la legislación que facilite el proceso de suspensión del consumo del tabaco. Cuando un agente de salud interviene en personas con toxicomanía, la posibilidad de éxito en el retiro del consumo se multiplica al menos en cinco veces, en contraste con quién no recibe apoyo y orientación calificada. ${ }^{8}$

La mujer, debería ser el centro de atención en la promoción de la salud desde el inicio de su edad 
reproductiva y cuando tenga la oportunidad de estar en contacto con un agente de salud sensibilizado y suficientemente capacitado para cumplir con esta labor, así como motivado a desarrollar investigación local. Debemos desarrollar métodos de consejería efectiva a la paciente y sus familias, que sean culturalmente aceptables y costo-efectivos, con participación de profesionales de la salud en todos los niveles. ${ }^{8}$ Además, se debe investigar en áreas como la evaluación del uso de incentivos para aumentar la cesación de tabaquismo; el uso de farmacoterapia como el bupropión, terapia de reemplazo con nicotina o uso de vareniclina, ya que no existe información y evidencia científica adecuada que permita recomendar su uso como adyuvantes en la reducción del tabaquismo durante el embarazo. ${ }^{9}$ La educación del núcleo familiar y no sólo a la gestante, así como el uso de intervenciones en el posparto, son áreas de investigación que permanecen inexploradas.

La información que tenemos acerca de las alteraciones perinatales resultantes de la exposición al tabaco y los productos del cigarrillo, está concentrada en el tabaquismo activo. Aquellas pacientes que son fumadoras pasivas (resultado de la exposición al uso del tabaco en su hogar o sitio de trabajo) escapan completamente de cualquier asesoría prenatal sobre la posibilidad de presentar morbilidad durante el embarazo como resultado de dicha exposición. ¿Qué tal imaginarnos, como una estrategia permanente, la inclusión en la clasificación de alto riesgo obstétrico a la fumadora pasiva? ¿Sensibilizaría esa condición a los proveedores de la salud? ¿Habría un impacto en la salud de los recién nacidos, a través de la reducción de la prevalencia de pacientes con ruptura temprana de membranas o RCIU, aunque desconociéramos la prevalencia real de estas condiciones mórbidas en las fumadoras pasivas?

Solo podremos determinar el impacto de las acciones de promoción o prevención si tenemos un diagnóstico epidemiológico de la situación actual, con estudios como el liderado por Cortés-
Yepes, conociendo la prevalencia de la condición, midiendo la asociación entre la condición de fumadora activa y pasiva con morbilidad perinatal, el porcentaje atribuible al uso del tabaco y cigarrillo y el riesgo atribuible poblacional, para luego, implementar medidas de salud pública de impacto y prevención.

Acompañando a Hernán Cortés-Yepes en su liderazgo y visión, la Red Mundial para la Investigación y el Desarrollo de la Salud Perinatal y Reproductiva, (www.gnprh.org), en la que participamos, ha iniciado una primera aproximación de trabajo de campo para conocer en varias instituciones de la ciudad de Medellín, tanto universitarias como de prestación de servicios de salud, la actitud, el conocimiento de la morbilidad perinatal y el acceso a herramientas para disminuir o evitar el hábito de fumar de los proveedores de salud quienes participan en la atención a la mujer embarazada. Nuestras observaciones iniciales (Britt Severson, MPH, observación personal), apoyadas por este trabajo novedoso de Cortés-Yepes, refuerzan las hipótesis planteadas en los párrafos anteriores y nos convocan a trabajar unidos para mejorar la prestación de mejores servicios a mujeres embarazadas, fumadoras activas, pasivas y sus familias.

Un período preconcepcional, un embarazo y un posparto, libres de la exposición activa o pasiva al tabaco y sus efectos nocivos, son vitales para la madre y el feto. Invitamos a los lectores de la revista a participar activamente en el desarrollo de estrategias que nos permitan prevenir esta epidemia emergente.

\section{EDITORES INVITADOS}

\section{Jorge E. Tolosa, M.D., MSCE}

Profesor Asociado de Obstetricia y Ginecología, Oregon Health \& Science University, Portland, Oregon, EE.UU. Red Mundial para la Investigación y el Desarrollo de la Salud Perinatal y Reproductiva (Global Network for Perinatal \& Reproductive Health). (www.gnprh.org). Portland, Oregon, (EE.UU). Correo electrónico: tolosaj@ohsu.edu 
Luis Guillermo Echavarría-Restrepo, M.D., MS Ginecoobstetra, Universidad Pontificia Bolivariana. Coordinador de Ginecología y Obstetricia Universidad Pontificia Bolivariana. Magister en Salud Pública Universidad de Antioquia. Magister en Epidemiología Universidad CES. Medellín (Colombia).

Correo electrónico: echavarrialuis@yahoo.es

\section{Bernardo Agudelo J, M.D., GO}

Epidemiólogo, Profesor del Departamento de Ginecología y Obstetricia, Coordinador Línea Salud Cardiovasculary Metabólica de la Mujer de Nacer-SSR de la Universidad de Antioquia. Medellín (Colombia). Correo electrónico: bernardo.agudelo@gmail.com

\section{REFERENCIAS}

1. Cnattingius S. The epidemiology of smoking during pregnancy: smoking prevalence, maternal characteristics, and pregnancy outcomes. Nicotine Tob Res 2004;6:125-40.

2. Gilliland FD, Berhane K, McConnell R, Gauderman WJ, Vora H, Rappaport EB, et al. Maternal smoking during pregnancy, environmental tobacco smoke exposure and childhood lung function. Thorax 2000;55:271-6.

3. WHO. Report on the Global Tobacco Epidemic, 2008. The MPOWER package. Geneva, Switzerland: World Health Organization; 2008.
4. Vélez-Gómez MP, Barros FC., Echavarría-Restrepo LG, Hormaza-Ángel MP. Prevalencia de bajo peso al nacer y factores maternos asociados: Unidad de Atención y Protección Materno Infantil de la Clínica Universitaria Bolivariana, Medellín (Colombia). Rev Colomb Obstet Ginecol 2006;57:264-70.

5. Bloch M, Althabe F, Onyamboko M, Kaseba-Sata C, Castilla EE, Freire S, et al. Tobacco use and secondhand smoke exposure during pregnancy: an investigative survey of women in 9 developing nations. Am J Public Health 2008;98:1833-40.

6. Klebanoff MA, Levine RJ, Clemens JD, DerSimonian R, Wilkins DG. Serum cotinine concentration and selfreported smoking during pregnancy. Am J Epidemiol 1998;148:259-62.

7. Mejía R, Martínez VG, Gregorich SE, Pérez-Stable EJ. Physician Counseling of Pregnant Women About Active and Second-hand Smoking in Argentina. Acta Obstet Gynecol Scand 2010;89:490-5.

8. Lumley J, Chamberlain C, Dowswell T, Oliver S, Oakley L, Watson L. Interventions for promoting smoking cessation during pregnancy. Cochrane Database Syst Rev 2009;(3):CD001055.

9. Oncken CA, Dietz PM, Tong VT, Belizán JM, Tolosa JE, Berghella V, et al. Prenatal tobacco prevention and cessation interventions for women in low- and middle-income countries. Acta Obstet Gynecol Scand 2010;89:442-53. 